echogenicity in 155, 15 and 19 cases, respectively. Sonographic findings were normal in 74 children. Among the 115 children with abnormal findings, the most common sonographic diagnosis was appendicitis, which was diagnosed in 84 children (26 of whom had renal cortex hyperechogenicity). No patients were diagnosed with renal disease or urinary tract infection. Serum creatinine levels and urine sediment in all tested patients with renal cortex hyperechogenicity were normal.

All but three patients with hyperechogenicity were re-examined at between 1 day and 1 year after sonography; on re-examination all acute abdominal complaints and all hyperechogenicity had resolved. One year after sonography, no child who had shown hyperechogenicity of the renal cortex showed evidence of a renal disorder.

Original article Wiersma F et al. (2008) Increased echogenicity of renal cortex: a transient feature in acutely ill children. AJR Am J Roentgenol 190: 240-243

\section{Renal length discrepancy on ultrasound predicts pediatric renal abnormalities}

In adults, $a \geq 10 \mathrm{~mm}$ difference in the lengths of the right and left kidneys on ultrasound is considered predictive of renal pathology. Khazaei et al. sought to determine a cutoff point at which renal length discrepancy (RLD) on ultrasound is predictive of an abnormality detected by technetium-99m-labeled dimercaptosuccinic acid (DMSA) scanning in children.

On ultrasound, 50 of 90 children (aged 1 week to 17 years) had a left kidney that was longer than the right-as is the case for most healthy individuals - and 33 children had a right kidney that was longer than the left. An abnormality (differential renal function $<45 \%$ and/or a renal scar) was detected by DMSA scanning in 40 patients. Mean RLD by ultrasound was significantly greater in children with abnormal DMSA scans than in those with normal DMSA scans $(12.4 \mathrm{~mm}$ vs $3.9 \mathrm{~mm} ; P<0.0001)$.

The cutoff ultrasound RLDs that resulted in the best positive predictive values for an abnormality on DMSA scan were $\geq 6 \mathrm{~mm}$ in children whose right kidney was longer than the left, and $\geq 10 \mathrm{~mm}$ for children whose left kidney was longer than the right (positive predictive values $100 \%$ and $79 \%$, respectively). When analyzed by age, similar cutoffs were found for children older than 48 months - the age at which renal growth slows. By contrast, children younger than 48 months should be referred for further evaluation when RLD (either left kidney longer than the right or vice versa) determined by ultrasonography exceeds $6 \mathrm{~mm}$.

Original article Khazaei MR et al. (2008) Renal length discrepancy by ultrasound is a reliable predictor of an abnormal DMSA scan in children. Pediatr Nephrol 23: 99-105

\section{Commonly used screening test fails to detect Fabry disease in some patients}

Early detection of Fabry disease is desirable, as accumulation of glycosphingolipids in the kidney and other tissues can be prevented. Andrade et al. assessed the performance of a fluorometric a-galactosidase assay, which can be used to screen for the disease, in an ethnically diverse cohort of male patients with CKD.

$\alpha$-Galactosidase activity data were assayed in the plasma of 499 patients from two hospitals in Vancouver, Canada who did not have known Fabry disease. The study population was $71 \%$ white, $15 \%$ Asian and $11 \%$ Southeast Asian. No new cases of Fabry disease were detected. When the assay was repeated in five patients who had among the lowest enzyme activity values $(1.6-2.5 \mathrm{nmol} / \mathrm{h}$ per $\mathrm{ml})$, four of these patients had results within the normal range $(3.0-8.1 \mathrm{nmol} / \mathrm{h}$ per $\mathrm{ml})$.

Results from a subset of patients who were tested for $\alpha$-galactosidase activity in leukocytes showed that the leukocyte assay is less variable than the plasma assay. Samples from three patients with known Fabry disease were analyzed with both the plasma and the leukocyte assays, and all three patients showed low enzyme activity in the leukocyte assay, but two had normal activity in the plasma assay. Use of an inhibitor of $\alpha$-galactosidase $B$ in the plasma assay did not affect the variability of the results. Clinicians should, therefore, be aware that screening for Fabry disease by use of the $\alpha$-galactosidase plasma assay can lead to false-negative results.

Original article Andrade J et al. (2008) Screening for Fabry disease in patients with chronic kidney disease: limitations of plasma $\alpha$-galactosidase assay as a screening test. Clin J Am Soc Nephrol 3: 139-145 